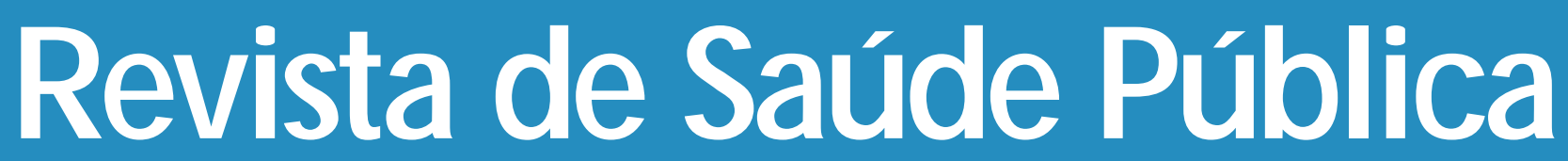

$\begin{array}{lllllll}\mathbf{J} & \mathbf{O} & \mathbf{U} & \mathbf{R} & \mathbf{N} & \mathbf{A} & \mathbf{L}\end{array}$

0 F

$\mathbf{P} \mathbf{U}$

B L I C

H $\quad$ E $\quad A \quad L \quad T \quad H$

\title{
Factores de riesgo para el desarrollo psicomotor en lactantes nacidos en óptimas condiciones biológicas*
}

\section{Risk factors for psychomotor development among infants born under optimal biological conditions}

Isidora de Andraca, Paulina Pino, Alicia de La Parra, Francisca Rivera y Marcela Instituto de Nutrición y Tecnología de los Alimentos. Universidad de Chile. Santiago, Chile (I.A., A. P., F.R., M.C.), Departamento de Salud Pública. Facultad de Medicina O riente, Universidad de Chile. Santiago, Chile (P. P.) 


\title{
Factores de riesgo para el desarrollo psicomotor en lactantes nacidos en óptimas condiciones biológicas*
}

\section{Risk factors for psychomotor development among infants born under optimal biological conditions}

\author{
Isidora de Andraca, Paulina Pino, Alicia de La Parra, Francisca Rivera y Marcela Castillo
}

Instituto de Nutrición y Tecnología de los Alimentos. Universidad de Chile. Santiago, Chile (I.A., A. P., F.R., M.C.), Departamento de Salud Pública. Facultad de Medicina O riente, U niversidad de Chile. Santiago, Chile (P. P.)

\begin{abstract}
Resumen
Objectivo Evaluar el efecto de factores de riesgo para el desarrollo infantil en niños, de nivel socioeconómico bajo, nacidos en óptimas condiciones biológicas pero expuestos a condiciones sociales adversas.

Metodología Lactantes de ambos sexos em número de 788, fueron exhaustivamente estudiados en términos de: alimentación, crecimiento, ambiente físico y psicosocial. Se controlaron prospectivamente hasta los 12 meses de edad, momento en que se evaluó el desarrollo mental (MDI) y motor (PDI) a través de la escala de Bayley. A partir de las variables medidas se identificó 18 factores de riesgo, los que fueron dicotomizados (bajo y alto riesgo).

Resultados Lactancia materna, temperamento del niño, inteligencia materna y estimulación en el hogar se asocian consistentemente con MDI y PDI. Luego de ajustar por covariables, la estimulación en el hogar persiste como factor significativo en la explicación de la variabilidad del MDI y PDI, en tanto que el sexo del niño se consolida como factor condicionante del desarrollo motor. El efecto acumulado de 7 o más factores de riesgo se asocia con una disminución significativa de los puntajes de desarrollo. La combinación de categorías de riesgo de temperamento infantil, inteligencia materna, rol del padre y estimulación en el hogar alcanza mejor predicción del desarrollo infantil que otras combinaciones puestas a prueba en este estudio.

Conclusione Se concluye que, aún en niños biológicamente indemnes, el desarrollo psicomotor se afecta negativamente ante la presencia de condiciones ambientales adversas que actúan simultáneamente.
\end{abstract}

Dessarrollo infantil. Desempeño psicomotor. Factores de riesgo.

\footnotetext{
* Estudio financiado por "National Institutes of Health".

Correspondencia para/Correspondence to: Isidora de Andraca - Casilla 138-11, Santiago , Chile E-mail: iandraca@uec.inta.uchile.cl

Recibido en 1.8.1996. Reapresentado en 8.9.1997. Aprobado en 29.9.1997.
} 


\section{Abstract}

Objective The objective of this study is to evaluate the effect of risk factors on infant development, among low socioeconomic children born under optimal biological conditions, and who are exposed to adverse social circumstances.

Method Infants of both sexes, in the amount of 788, were studied and controlled prospectively at between 6 and 12 months. Their mental (MDI) and psychomotor (PDI) development was evaluated using the Bayley Scale of Infant Development. Eighteen risk factors were identified and dichotomized (high or low risk).

Results Breast feeding, child temperament, maternal intelligence and home stimulation are consistently associated with lower MDI and PDI. After adjustment for covariables, home stimulation persisted as a significant factor for explaining the variation of both developmental indexes and child sex also appears as a risk factor for motor skills development. The accumulated effect of 7 or more risk factors is associated with a significant decrease of development scores. The combination of risk categories of child temperament, maternal IQ, paternal rolle and home stimulation shows higher prediction power for infant development than other combinations analyzed in this study.

Conclusion The findings suggest than even for children born under optimal biological conditions their psychomotor development is negatively affected by the presence of simultaneous adverse environmental conditions.

Child development. Psychomotor performance. Risk factors.

\section{Resumo}

Objetivo Avaliar o efeito dos fatores de risco para o desenvolvimento infantil em crianças de nível socioeconômico baixo, nascidos em ótimas condições biológicas, mas expostos a condições sociais adversas.

Metodologia

Lactentes dos dois sexos, no total de 788 , foram exaustivamente estudados quanto a alimentação, crescimento, ambiente físico e psicossocial controladas. Controlaram-se prospectivamente até os 12 meses de idade, momento em que se avaliou o desenvolvimento mental (MDI) e o motor (PDI) através da escala de Bayley. A partir das variáveis medidas, identificaram-se 18 fatores de risco, que foram dicotomizados (baixo e alto risco).

Resultados Amamentação materna, temperamento da criança, inteligência materna e estímulo no lar associam-se consistentemente com MDI e PDI. Após ajuste por co-variáveis, o estímulo no lar persiste como fator significativo na explicação da variabilidade do MDI e do PDI, e o sexo da criança aparece-se como fator condicionante do desenvolvimento motor. O efeito acumulado de sete ou mais fatores de risco associa-se a uma diminuição significativa das pontuações de desenvolvimento. A combinação de categorias de risco de temperamento infantil, inteligência materna, papel do pai e estímulo no lar, atinge melhor predição do desenvolvimento infantil que outras combinações analisadas.

Conclusão Conclui-se que, mesmo para as crianças em ótimas condições biológicas, o desenvolvimento psicomotor é afetado na presença de condições ambientais adversas que agem simultaneamente.

Desempenho psicomotor. Desenvolvimento infantil. Fatores de risco. 


\section{INTRODUCCIÓN}

El primer año de vida se caracteriza por una explosión de habilidades motoras y cognitivas, cuya secuencia y momento de aparición son dependientes de factores genéticos y madurativos. La adquisición de nuevas habilidades se basa en un adecuado desarrollo prenatal, el que ejerce una fuerte influencia sobre el curso del desarrollo postnatal, estableciendo las bases biológicas para un desarrollo normal. Si las condiciones genéticas y prenatales han sido favorables, el niño contará con un potencial de resiliencia ante adversidades físicas o psicológicas, en especial si las condiciones de crianza son razonablemente apoyadoras. Por el contrario, una constitución genética adversa o un cuidado prenatal insuficiente establecen bases para la vulnerabilidad del desarrollo. Dependiendo de la severidad de la condición adversa, el desarrollo puede verse afectado irreversiblemente o puede presentar sólo una alteración, logrando finalmente alcanzar las metas evolutivas (Kopp y Kaler ${ }^{20}$, 1989).

Cuando un niño presenta indemnidad biológica, el desarrollo temprano sigue un curso preestablecido en que sus hitos fundamentales (cognición, lenguaje, motricidad) difícilmente se ven alterados. Un medio ambiente favorable puede facilitar un desarrollo normal, el cual posibilita una mejor exploración e interacción con su entorno. Por el contrario, un ambiente desfavorable puede enlentecer el ritmo del desarrollo, lo que disminuiría la calidad de la interacción del niño con su medio, restringiendo su capacidad de aprendizaje (Kopp y McCaal ${ }^{19}$, 1982; Horowitz ${ }^{17}, 1988$ ).

La literatura actual ha enfatizado la importancia de los factores de riesgo biológicos y sociales sobre el curso del desarrollo infantil. Prematuridad, bajo peso de nacimiento, malnutrición, padres adolescentes o añosos, madre soltera, ausencia del padre, depresión materna, bajo nivel educacional y/ o problemas psiquiátricos de los padres son algunos de los factores de riesgo que se relacionan con el desarrollo psicológico infantil $1^{6,15,16,21,23,25,27,35}$. Tales factores de riesgo generalmente no ocurren en forma aislada; a medida que se combina un mayor número aumenta la probabilidad de un menor rendimiento cognitivo. Además, estos factores son más frecuentes en condiciones de pobreza, postulándose que el nivel socioeconómico bajo amplifica la vulnerabilidad biológica $^{6,18,29}$.
Diferentes aspectos confluyen para que el primer año de vida tenga una especial importancia desde el punto de vista del desarrollo infantil. En primer lugar, el sistema nervioso central se encuentra en una fase de crecimiento rápido y es aún muy vulnerable a las influencias del medio (Dobbing ${ }^{14}, 1968$ ). Además, el establecimiento de vínculos afectivos estables y seguros sientan las bases del desarrollo socio-emocional (Sameroff $\left.{ }^{29}, 1986\right)$. Por otra parte, nuevas habilidades motoras generan un cambio en la posición referencial en el espacio, lo que permite la adquisición de otras habilidades (Pollitt ${ }^{26}, 1994$ ). Por último, las habilidades cognitivas y lingüísticas crecientes posibilitan la comprensión y organización progresiva del entorno. Por todo lo anterior, la presencia de factores de riesgo para el desarrollo psicomotor-al interrumpir estos procesosdisminuye las potencialidades del niño.

$\mathrm{Si}$ bien se ha descrito el efecto negativo de factores de riesgo sobre el desarrollo de niños biológicamente vulnerables, se desconoce el impacto de condiciones adversas sobre el desarrollo de niños que biológicamente indemnes ${ }^{28,31,32}$.

El objetivo de lo presente estudio es cuantificar el efecto de factores de riesgo sobre el desarrollo mental y motor en una población de lactantes, nacidos en óptimas condiciones biológicas y sin enfermedades de importancia durante el primer año de vida. Estos niños ofrecen una oportunidad única de observar el impacto de condiciones variables de pobreza sobre el desarrollo infantil.

\section{METODOLOGÍA}

Los datos presentados fueron recolectados durante un estudio realizado en Santiago de Chile a partir de 1991. Su objetivo central fue prevenir la anemia por deficiencia de hierro a través de la fortificación de la dieta infantil. De acuerdo a un diseño randomizado y prospectivo, lactantes de 6 meses* fueron aleatoriamente asignados a recibir una fórmula con alto contenido de hierro $(12 \mathrm{mg} /$ L) o fórmula con menor contenido de hierro (mínimo 1,5 $\mathrm{mg} / \mathrm{L}$; promedio $2,3 \mathrm{mg} / \mathrm{L}$ ) hasta el año de edad. Este estudio permitió una completa medición de variables del niño y del medio ambiente social y familiar.

\section{Sujetos}

Este análisis incluye 788 lactantes, que corresponden a los niños incluidos en el estudio entre 1991 y 1994. Se invitó a participar a todos los niños de cuatro meses que

* Este estudio fue aprobado por los comités de ética del INTA, Universidad de Chile y del Centro de Desarrollo y Crecimiento Humano de la Universidad de Michigan, USA. 
cumplieran con los siguientes requisitos: peso de nacimiento $>3.000 \mathrm{gr}$, parto único vaginal o cesárea programada, sanos* y que no asistieran a sala cuna. Se definió además como criterio de entrada otras condiciones que facilitaban el manejo del estudio: domicilio dentro del área de los consultorios y presencia de cuidadora estable alfabetizada. Para evitar interferir con la lactancia materna, sólo se invitó a participar a niños que a los 6 meses de edad ya hubieran iniciado espontáneamente la alimentación láctea artificial (al menos $250 \mathrm{cc}$ de leche de vaca o fórmula/día), ya que la fortificación se realizó a través de una fórmula con alto contenido de hierro. El cumplimiento de estos criterios fue confirmado en dos visitas domiciliarias, después de las cuales se obtuvo el consentimiento informado de los padres de los niños elegibles.

Un total de 4.351 lactantes de 4-6 meses de edad fueron contactados en los consultorios, de los cuales 1.549 cumplían todos los criterios de entrada de acuerdo a la información entregada por la madre. Los padres de 1.486 de estos niños $(95,9 \%)$ mostraron interés en participar en el estudio. Durante las visitas al hogar se descartó $11,7 \%$ de los niños (n:174), por incumplimiento de criterios de entrada. De los que calificaban, 94,3\% (n:1.237) aceptaron participar. Se excluyó 34 niños por presentar anemia por defi-ciencia de hierro a los 6 meses de edad (Hemoglobina $<102 \mathrm{~g} / \mathrm{L}$ ), los que fueron tratados con hierro oral. Igualmente se excluyó 34 niños con niveles adecuados de hemoglobina, que fueron invitados a participar como controles de los anémicos en otro protocolo.

La muestra final ingresada al estudio durante 19911994 estuvo constituida por 1.169 lactantes, de los cuales $27,9 \%$ (n:326) no completaron el protocolo. Las causas de exclusión más frecuentes fueron incumplimiento de protocolo (n:102)*, cambios de domicilio fuera del sector (n:67), hospitalización del niño >7 días (n:39), rechazo de leche (n:19), cuidadora no permanente (n:15) y madre trabaja fuera del hogar con el niño (n:16). De los 843 niños que completaron el estudio, 788 (93,5\%) contaban con información completa de desarrollo psicomotor a los 12 meses de edad, los que se incluyen en este análisis.

\section{Evaluaciones}

Cada niño recibió atención mensual de control sano y atención de libre demanda en morbilidad, ambas realizadas por un pediatra del proyecto. Ello permitió registrar confiablemente información de episodios mórbidos y de crecimiento, (peso, talla circunferencia craneana) (Tablas de referencia de NCHS-National Center for Health Statistics ${ }^{24}$, 1978). Encuestadoras de terreno visitaron semanalmente los hogares consignando consumo de leche y morbilidad intercurrente. A los 12 meses se realizó además una punción venosa para la evaluación del estado hematológico.

\section{Mediciones Conductuales y del Desarrollo}

A los 7 meses de edad se evaluó temperamento del niño, a través del Cuestionario de Temperamento Infantil de Bates (ICQ para 6 meses) (Bates³, 1979, Bates $^{4}$, 1988). Este instrumento se basa en la percepción materna del comportamiento de su hijo(a) y consta de 24 ítems, entregando resultados de acuerdo a cuatro factores: dificultad, apagamiento, impredecibilidad y no adaptabilidad.

El desarrollo psicomotor se evaluó a los 12 meses de edad a través de la Escala del Desarrollo Infantil de Bayley, que entrega un Indice de Desarrollo Mental (MDI) y un Indice de Desarrollo Motor (PDI). Cuenta además con una Escala de Registro de Conducta (Bayley ${ }^{5}$, 1969).

\section{Evaluación del Ambiente del Niño}

Cuando el niño tenía aproximadamente 6 meses de edad se recolectó en el hogar información básica del nivel socioeconómico (Medición de Indice Específico), complementada con información de composición familiar, educación y ocupación de los padres, consumo de alcohol y otras variables sociodemográficas generales (Alvarez et al. $\left.{ }^{1}, 1982\right)$.

La calidad de la estimulación, en el hogar se midió a través del inventario HOME (Home Observation for Measurement of the Environment) para 0-3 años de edad (Caldwell $\left.{ }^{9}, 1975\right)$. El HOME es una escala construida para detectar indicadores de la cantidad y calidad del apoyo social, emocional y cognitivo disponible para el niño en su hogar. Ha sido ampliamente utilizado y ha resultado ser sensible a la detección de características familiares al ser aplicada en poblaciones latinoamericanas ${ }^{10,11,22}$. Esta medición fue realizada durante una visita sorpresa al hogar cuando el niño tenía 10 meses de edad.

La inteligencia materna fue estimada a través de una versión abreviada del WAIS, elaborada a partir de un estudio de campo con mujeres chilenas de nivel socioeconómico bajo, similar a la muestra de este estudio (De Andraca et al. ${ }^{12}$, 1993). A los 7 meses de edad del niño se aplicó el Cuestionario de Animo del C-DES, para identificar madres con síntomas depresivos (Devins y Orme ${ }^{13}$, 1985). Por último, se obtuvo una medida de eventos estresantes, a través de un listado especialmente confeccionado para este estudio, que incluye problemas económicos, de salud, de relación con otras personas (pareja, familiares, vecinos), enfermedades crónicas, problemas con la justicia, nuevo embarazo de la madre, entre otros. Este instrumento entrega un puntaje que varía entre 0 y 29 , que corresponde, de acuerdo a la información entregada por la madre, a la sumatoria de eventos estresantes presentes cuando el niño tenía aproximadamente 11 meses de edad.

Todo el personal fue especialmente entrenado y estandarizado en la realización de las mediciones.

\footnotetext{
* Libres de anormalidades congénitas, complicaciones neonatales, ictericia con requerimiento de fototerapia, hospitalizaciones previas o enfermedades crónicas.

** Por ejemplo: no asistir al consultorio en 3 citaciones consecutivas, no estar presente en el horario convenido para las visitas semanales al hogar en 3 oportunidades consecutivas, no aceptar la toma de muestra de sangre, entre otros.
} 


\section{Definición de Factores de Riesgo para el Desarrollo Psicomotor}

A partir de las mediciones de variables biológicas y psicológicas del niño y de las variables ambientales disponibles se identificó 17 factores de riesgo para el desarrollo. Los factores fueron elegidos de acuerdo a dos criterios: la existencia de antecedentes en la literatura que mostraran su relación con el desarrollo infantil, y la confiabilidad en el nivel de la medición. Los niveles de riesgo fueron establecidos mediante dicotomización de las variables, orientada por criterios conceptuales en aquellos factores en que existieran antecedentes para establecer claramente un punto de corte. Las variables de temperamento infantil, nivel socioeconómico, número de orden, eventos estresantes, depresión materna e ingesta de alcohol fueron dicotomizados de acuerdo a criterio estadístico, definiéndose como riesgo el $25 \%$ con condiciones más adversas para el desarrollo infantil (Tabla 1).

\section{Análisis de D atos}

Las diferencias entre los grupos en las variables continuas fueron evaluadas a través de análisis de varianza. Las variables discretas fueron comparadas a través de la prueba de Chi cuadrado. Dado que las variables dependientes MDI y PDI son continuas, el análisis multivariado se realizó a través del método de regresión múltiple, utilizando el procedimiento de ingreso progresivo de variables en el modelo (método stepwise). Todos los análisis fueron realizados computacionalmente, utilizándose el paquete estadístico SPSS, definiéndose un nivel alfa de ,05 para las pruebas de significación estadística.

\section{RESU LTAD O S}

\section{Características G enerales de la Muestra}

La muestra está compuesta por niños de ambos sexos (48\% varones), con peso de nacimiento de $3.509 \pm 364,5$ gr. y talla de nacimiento de 50,5 $\pm 1,7$ $\mathrm{cm}$. La totalidad de los niños recibió lactancia materna; con una duración promedio de 74,3 \pm 55 días.

El nivel socioeconómico promedio corresponde a la categoría bajo-alto del instrumento Indice Específico $(40,3 \pm 7,9)$. Ninguna de las familias incluidas alcanza el nivel más bajo (miseria); $26 \%$ de la muestra se encuentra en el rango medio-bajo. El trabajo paterno es inestable en $41 \%$ de los casos ( $8 \%$ cesantes, $33 \%$ trabajo ocasional); $29 \%$ presenta trabajo calificado y estable. El 94,2\% de los niños son cuidados por sus propias madres y en el resto lo hace una cuidadora estable; $82,6 \%$ de los niños viven con ambos padres, existiendo sólo 8,6\% de abandono paterno completo, es decir el padre no mantiene ningún tipo de vínculo con el niño. El número de orden de nacimiento promedio es $2,1 \pm 1,1$. De hecho, $70,7 \%$ de las familias tienen 1-2 hijos, 20,1\% 3 y sólo 9,2\% tienen cuatro o más.

La educación materna promedio es de 9,4 \pm 2,7 años completos cursados, destacándose que sólo 37,8\% no supera el nivel básico de educación. Los padres presentan un nivel de educación levemente inferior $(8,7 \pm 3,3), 45,6 \%$ completó

Tabla 1 - Definición de factores de riesgo del desarrollo psicomotor.

Table 1 - Definition of risk factors for psychomotor development.

\begin{tabular}{lccc}
\hline Variable de riesgo & Sin riesgo & Riesgo alto & Punto de corte \\
\hline Sexo & Femenino & M asculino & - - \\
Peso de nacimiento & $\mathrm{z} \geq 0$ & $\mathrm{z}>0$ & $\mathrm{z}=0$ \\
Lactancia materna & $\geq 30$ días & $<30$ días & 30 días \\
$\quad$ Temperamento del niño & $75 \%$ superior & $25 \%$ inferior & 23 \\
$\quad$ Dificultad & $75 \%$ superior & $25 \%$ inferior & 10 \\
$\quad$ Apagamiento & $75 \%$ superior & $25 \%$ inferior & 11 \\
$\quad$ Impredictibilidad & $75 \%$ superior & $25 \%$ inferior & 15 \\
$\quad$ No adaptable & $75 \%$ superior & $25 \%$ inferior & $z=-0,88$ \\
z talla 12 meses & $75 \%$ superior & $25 \%$ inferior & 45 \\
Nivel socioeconómico & Vive con el niño & No vive con el niño & -- \\
Presencia del padre & $75 \%$ superior & $25 \%$ inferior & $\geq 3$ hijos \\
Número de hijos & $75 \%$ superior & $25 \%$ inferior & 5 factores \\
Stress familiar & $\geq 6$ años & $<6$ años & -- \\
Educación padre & $\geq 8$ años & $<8$ años & -- \\
Educación madre & Cl $>80$ & $25 \%$ inferior & -- \\
Inteligencia materna & $75 \%$ superior & $25 \%$ inferior & 25 \\
Depresión materna & $75 \%$ superior & 10 más bebedores & 27 \\
Estimulación en el hogar & 0 bebedores & -- \\
Ingestión de alcohol & &
\end{tabular}


la básica, 51,3\% la media, y 3,3\% habían iniciado la educación superior.

En cuanto a las variables microambientales, la inteligencia materna corresponde en promedio al nivel "normal lento" (CI: 83,9 \pm 9,8). El 30,5\% de las madres obtuvo un $\mathrm{CI}<80$, valor aceptado como punto de corte para un funcionamiento intelectual insuficiente. El ánimo materno durante la última semana corresponde a distress psicológico en $46 \%$ de las mujeres. Las familias están expuestas a 4,7 $\pm 2,8$ eventos estresantes a partir del nacimiento del niño, $51,7 \%$ de las familias reportan presentar simultáneamente 4 o más eventos estresantes. La estimulación en el hogar, medida por la escala HOME, corresponde a percentil $50(30 \pm 4,8)$, similar a los datos normativos de la escala en USA $(30,4 \pm 7,7)$. El $32 \%$ de la muestra reporta que 1 o más miembros de la familia ingerían alcohol en forma habitual.

Los factores del temperamento infantil son normales de acuerdo a normas norteamericanas de la prueba de Bates, pero los puntajes promedio obtenidos por los niños de la muestra son levemente más altos en cada uno de los factores medidos. (Dificultad: 19,8 \pm 5,6, Apagamiento: 7,7 \pm 2,9, No Adaptabilidad: 11,7 \pm 4,9; Impredecibilidad: $8,6 \pm 3,0)$.

A los 12 meses de edad el crecimiento de los niños está dentro de límites adecuados ( $\mathbf{z}$ peso para la edad: -,32 \pm ,94; $\mathbf{z}$ talla edad: -,32, $\pm 94 ; \mathbf{z}$ peso para la talla: ,40 \pm ,9), destacándose que, tal como ha sido descrito anteriormente en la población chilena, el promedio de talla se sitúa bajo la mediana del estándar NCHS.

La evaluación del desarrollo psicomotor muestra puntajes promedios normales (MDI: 103,8 \pm 12,9; PDI: $96,7 \pm 15)$. Es llamativo que, de acuerdo a los datos normativos originales de la Escala de Bayley, sólo 5,9\% de los niños muestra puntajes inferiores a una desviación estándar en el MDI, en tanto que para el PDI la proporción se eleva a $17,1 \%$.

\section{D esarrollo Psicomotor y Factores de Riesgo}

El análisis de los promedios de MDI y PDI en cada categoría de los factores de riesgo muestra la existencia de asociaciones significativas (Tabla 2). El sexo del niño, que no fue definido inicialmente como factor de riesgo para el desarrollo, muestra una asociación significativa para el MDI; siendo que los varones obtienen puntajes inferiores. De acuerdo a este resultado, la variable sexo del niño fue incorporada a los análisis multivariados, definiéndose sexo masculino como categoría de riesgo.

Se realizó un análisis multivariado, a través del método de regresión múltiple, incorporando en forma lineal todas las variables definidas como factores de riesgo, e incluyendo además el sexo del niño. La variable nivel socioeconómico fue ingresada forzadamente en el primer lugar. Los resultados señalan que el modelo total permite explicar sólo $4,3 \%$ de la variabilidad del MDI. La única variable que permanece en el modelo es nivel de estimulación en el hogar (B:,561736; BETA: ,208671, p:,0002). Para el PDI el poder explicativo del modelo es de $6,6 \%$ y las variables que permanecen en la ecuación son estimulación en el hogar (B:,722549, BETA: ,237234; p<,0000; y sexo del niño (B:-3,233080; BETA: 1,$594064 ; \mathrm{p}<0,04)$, siendo sexo masculino

Tabla 2 - Valores de lo indice de desarrollo mental (MDI) y indice de desarrollo motor (PDI) a los 12 meses según variables de riesgo.

Table 2 - MDI and PDI values at 12 months by risk variables.

\begin{tabular}{lccc}
\hline Variable de riesgo & Riesgo bajo & Riesgo alto & $p<*$ \\
\hline MDI & & &, 001 \\
Sexo & $105,2 \pm 13,1$ & $102,4 \pm 12,5$ &, 01 \\
Lactancia materna & $104,6 \pm 12,7$ & $102,1 \pm 13,4$ &, 003 \\
Apagamiento & $104,5 \pm 12,9$ & $102,3 \pm 13,2$ &, 001 \\
Inteligencia materna & $105,1 \pm 12,4$ &, 002 \\
Estimulación hogar & $105,3 \pm 12,6$ & $101,1 \pm 13,9$ &, 002 \\
Ingestión de alcohol & $104,9 \pm 12,9$ & $99,6 \pm 13,8$ &, 05 \\
PDI & & $101,8 \pm 13,7$ &, 008 \\
Lactancia materna & $97,5 \pm 14,4$ & &, 005 \\
Apagamiento & $94,2 \pm 15,7$ & $95,2 \pm 15,3$ &, 05 \\
Nivel socioeconómico & $97,9 \pm 14,8$ & $97,4 \pm 14,6$ &, 03 \\
Presencia del padre & $97,9 \pm 14,8$ & $95,4 \pm 15,1$ &, 000 \\
Inteligencia materna & $97,4 \pm 14,9$ & $93,3 \pm 16,9$ & \\
Estimulación hogar & $98,6 \pm 14,2$ & $94,9 \pm 15,4$ & $91,4 \pm 16,6$ \\
*Análisisde Varianza & & &
\end{tabular}


una condición favorable para el desarrollo motor.

\section{Análisis Acumulativo}

El análisis multivariado permitió explicar sólo 4$6 \%$ de la variabilidad de los índices de desarrollo, resultado que no parece concordar con información consistente del efecto de variables ambientales sobre el desarrollo infantil.

Otra forma de abordar el problema es el cálculo de un puntaje de riesgo acumulativo de acuerdo al número de factores presentes en cada niño. El rango de factores de riesgo a que los niños estaban expuestos varió entre 0 y 12 , con un promedio de $4,3 \pm 2,2$. Es interesante resaltar que sólo el 1,3\% de los niños estaban expuestos a ningún factor de riesgo y $9,0 \%$ solamente a uno, en tanto que $26,9 \%$ estaban expuestos a 6 o más. El puntaje de riesgo acumulativo muestra valores de correlación moderados, pero consistentemente negativos, tanto con el MDI (r:,15, $\mathrm{p}<, 01)$ como con el PDI (r:-,25; $\mathrm{p}<, 001)$. Se comparó los promedios de MDI y PDI de acuerdo a categorías de riesgo, observándose una gradiente de disminución en ambos a medida que aumenta el número de factores de riesgo presentes en forma simultánea. El efecto se hace significativo para el MDI a partir de 5 o más factores, en tanto que para el PDI a partir de 7 (Prueba de Scheffe, p<,05) (Figura 1).

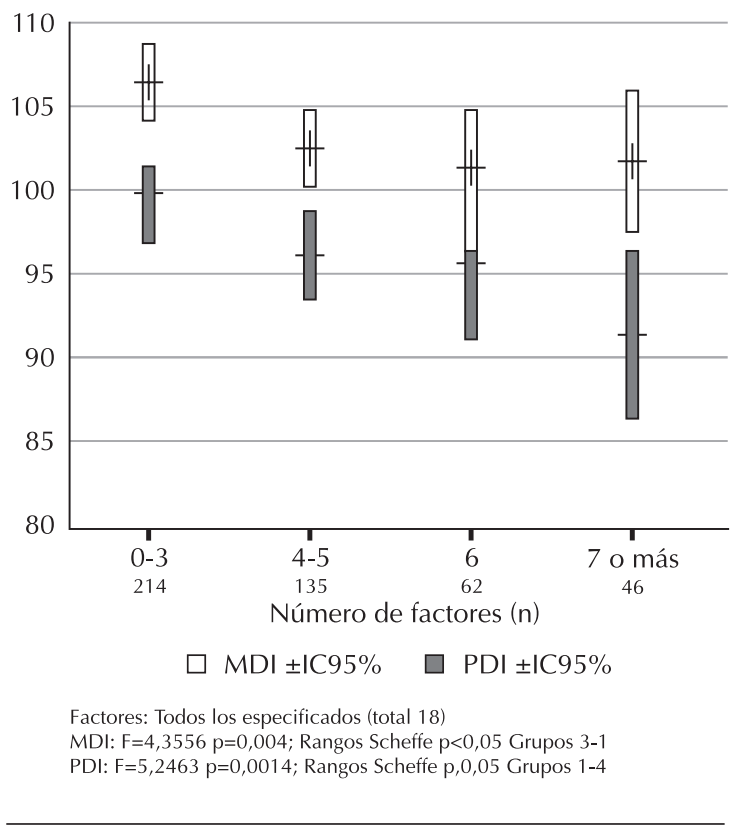

Figura 1- Efecto de factores de riesgo sobre el desarrolo psicomotor.

Figure 1- Cumulative effect of risk factors upon psychomotor development.
Se exploró distintas combinaciones de factores de riesgo como una manera de mejorar el poder discriminativo. En primer lugar se analizó el efecto acumulado de los factores que resultaron significativos en el análisis multivariado-estimulación en el hogar y sexo del niño-y se agregó la variable duración de lactancia materna exclusiva por existir consistente información en cuanto a su efecto sobre el desarrollo cognitivo. Los puntajes de MDI y PDI muestran una disminución significativa entre los niños que presentan hasta uno de estos factores de riesgo, y los que presentan 2 o 3 de ellos (Figura 2).

Se eligió además otras combinaciones de factores de riesgo, utilizando como criterio para seleccionarlos el que tuvieran un efecto significativo sobre el MDI y PDI en el análisis univariado, y que representaran una condición del niño, una del ambiente cercano y otra del ambiente social. La combinación más adecuada resultó ser la que incluye las variables estimulación en el hogar, presencia del padre, rendimiento intelectual de la madre y apagamiento del temperamento del niño (figura 2). El MDI presenta una disminución de 10,2 puntos entre los niños que no han estado expuestos a ninguno
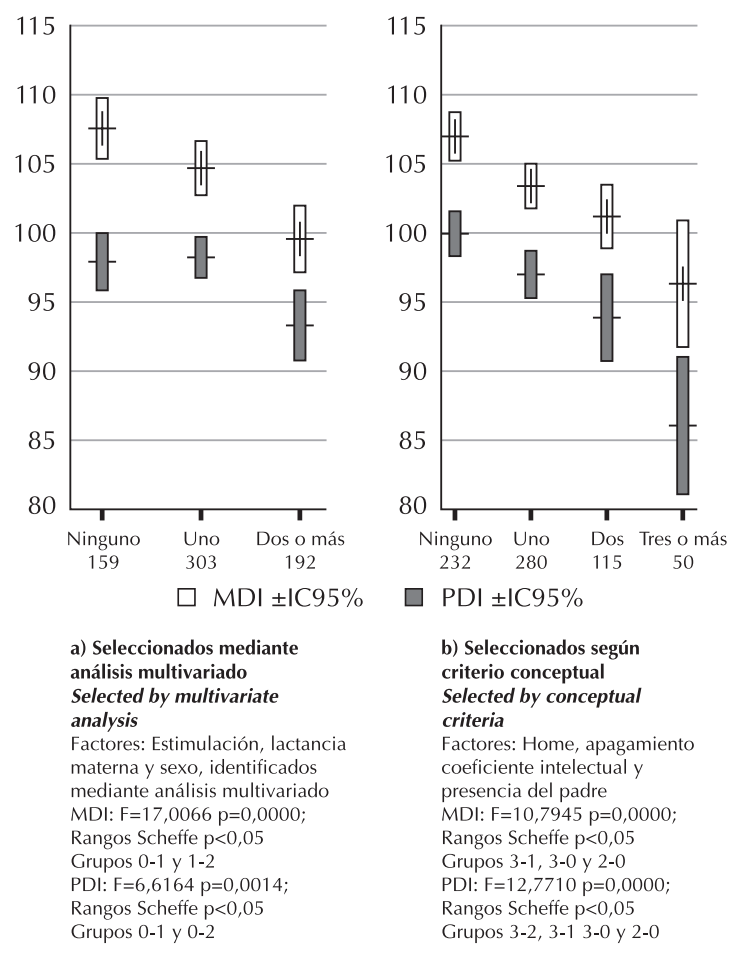

Figura 2- Efecto acumulado de factores de riesgo seleccionados sobre el desarrollo psicomotor. Figure 2 - Cumulative effect of risk factors upon psychomotor development. 
de estos cuatro factores, y los niños expuestos simultáneamente a todos ellos. La variación del PDI es de 13,5 puntos.

\section{DISCUSION}

Los estudios recientes del desarrollo infantil enfatizan la importancia de examinar el contexto en que éste ocurre, en especial el efecto de la presencia simultánea de múltiples factores de riesgo, tanto biológicos como ambientales. Más aún, la acción coincidente de estos factores debe ser analizada en presencia de factores protectores capaces de atenuar sus consecuencias potencialmente adversas.

Este estudio muestra que el desarrollo psicomotor de niños biológicamente indemnes se ve afectado por factores adversos durante el primer año. Tal efecto es menor que el descrito en niños biológicamente vulnerables, pero es suficiente para generar desventajas psicosociales capaces de perturbar el desarrollo ${ }^{28,31,32}$.

El hecho que los niños evaluados en este estudio sean altamente resilientes desde un punto de vista biológico permite dimensionar el impacto de variables socioambientales sobre el desarrollo infantil. Resulta llamativo que, a pesar de un desarrollo prenatal normal, los niños comienzan a deteriorar su crecimiento ya desde el primer año de vida. Este hecho, reportado anteriormente en la población chilena, muestra el efecto progresivo de variables socioeconómicas, ya que el déficit de crecimiento lineal ( $<1 \mathrm{DS})$ aumenta durante la niñez, alcanzando a $22 \%$ en el nivel socioeconómico medio y bajo ${ }^{2,34}$.

El presente estudio confirma, una vez más, que los niños de poblaciones desventajadas están expuestos a múltiples factores de riesgo simultáneos. Un alto porcentaje de niños registró la presencia de 5 o más factores de riesgo $(43,1 \%)$. A pesar de las condiciones adversas, el puntaje promedio de desarrollo mental no muestra deterioro, observándose apenas 5,9\% de niños que alcanzan rendimientos inferiores a una desviación estándar bajo el promedio. Postulamos que el buen rendimiento en habilidades mentales correspondería al efecto protector de un desarrollo prenatal adecuado y de condiciones neonatales altamente favorables. En cambio, 17,1\% de los niños muestra un puntaje de desarrollo motor menor que una desviación estándar bajo el promedio. Aparentemente, los niños en estudio serían menos resilientes al efecto adverso del ambiente sobre el desarrollo motor, pero alternativamente también podría plantearse interrogantes acerca de diferenci- as culturales en pautas de crianza.

Otro resultado concordante con la literatura es el efecto adverso de factores de riesgo sobre el desarrollo infantil, dependiendo del número de ellos presentes en forma simultánea (Sameroff et al ${ }^{29} 1987$; Sameroff et al. ${ }^{30}$ 1993). El puntaje promedio en habilidades mentales de niños menos expuestos (hasta tres factores de riesgo) es 4,2 puntos más alto que en los niños más expuestos (siete o más factores). En las habilidades motoras el efecto es más marcado, elevándose esta diferencia a 8,1 puntos. El desarrollo motor parece además más sensible que el desarrollo mental a los factores de riesgo en etapas tempranas. Sería necesario estudiar si este efecto se mantiene en edades posteriores, o si más adelante las habilidades cognitivas se hacen también más vulnerables a las deficiencias del medio ambiente.

El efecto adverso de la presencia simultánea de factores de riesgo para el desarrollo psicomotor aumenta cuando se considera factores de riesgo específicos, de mayor impacto sobre el desarrollo infantil: los niños de temperamento apagado, cuyas madres presentan coeficiente intelectual $<80$, con bajo nivel de estimulación en el hogar y con abandono paterno, muestran, en promedio, un índice de desarrollo mental 10,3 puntos más bajo y un desarrollo motor 13,5 puntos inferior que el de los niños que no están expuestos a riesgo en cada uno de esos factores. Lo anterior tiene implicancias en la planificación de acciones preventivas de apoyo al desarrollo, ya que con excepción del HOME—que requiere observación directa en el hogar-los otros tres factores mencionados son identificables durante los controles de salud, permitiendo reconocer niños en riesgo de enlentecimiento de su desarrollo psicomotor.

La estimulación en el hogar es la variable de mayor impacto sobre el desarrollo infantil. Esta variable, evaluada a través de la escala HOME, representa una medida del ambiente en que el niño se desarrolla, cuantificando el apoyo social, emocional y cognitivo. En este estudio las sub-escalas del HOME de mayor impacto son las relacionadas con el entorno afectivo del niño: responsividad de la madre a las necesidades del niño y la capacidad de la madre para involucrarse con el niño tienen efecto significativo sobre el rendimiento, tanto en las habilidades motoras como en las mentales. Estos resultados reafirman la necesidad de modificar las prioridades de intervención en el desarrollo infantil, enfatizando los programas de apoyo a la relación madre-hijo que serían más efectivos que los tradicionales programas de ejercitación 
psicomotora (Wasik et al. ${ }^{33}, 1991$ ).

El efecto de la variable Apagamiento del temperamento infantil plantea la posible contribución del niño como protagonista del medio en que se desarrolla. Como es sabido, durante largo tiempo este fue considerado un ser pasivo, sujeto al impacto de las condiciones que lo rodeaban. Actualmente se reconoce la influencia circular de las características conductuales del niño sobre las personas de su entorno, las que a su vez generan respuestas en sus cuidadores. La observación de un menor índice de desarrollo mental y motor en los niños de temperamento apagado conduce a plantear algunas preguntas. Es el Apagamiento del niño una variable que genera menores demandas de estimulación en sus cuidadores? Ejerce el Apagamiento infantil un efecto desmotivador para sus cuidadores, ofreciéndole menores oportunidades de estimulación? Es el Apagamiento una variable que pueda influir sobre el evaluador del desarrollo psicomotor, limitándo las oportunidades del niño para

\section{REFEREN CIAS}

1. ALVAREZ, M.L.; WURGAFT, F.; SALAZAR, M.E. Mediciones del nivel socioeconómico bajo urbano en familias con lactante desnutrido. Arch. Latinomer. Nutr., 32: 651-62, 1982.

2. AMIGO, H.; BUSTOS, P.; RADRIGÁN, M.E.; URETA, E. Estado nutricional en escolares de nivel socioeconómico opuesto. Rev. Med. Chile, 123: 1063-70, 1995.

3. BATES, J.E. Measurement of infant difficultness. Child Dev., 50: 7694-803, 1979.

4. BATES, J.E. Temperament in infancy. In: Osofsky, J.D. ed. Handbook of infant development. 2nd ed. New York, Willey, 1987. p. 1101-49.

5. BAYLEY, N. Bayley scales of infant development. New, York. Psychological Corporation, 1969.

6. BERNSTEIN, V.J.; HANS, S.L.; PERCANSKY, C. Advocating for the young child in need through strengthening the parent-child relationship. J. Clin. Child Psychol., 20: 28-41, 1991.

7. BRADLEY, R.H.; WHITESIDE, L.; MUNDFROM, D. Early indications of resilience and their relation to experience in the home environment of low birthweight, premature children living in poverty. Child Dev., 65: 346-60, 1994.

8. BRAZELTON, T.B. Importance of early intervention. In: Hibbs, E. D. ed. Children and families: studies in prevention and intervention. Madoison. International Universities Press, 1988. p. 107 - 20.

9. CALDWELL, B.M. Instruction manual: home inventory for infants. Rev. ed., Little Rock, University of Arkansas, Center la realización de las tareas? O es el Apagamiento del niño un marcador de una condición preexistente?

Otro elemento de interés es el efecto de la presencia del padre sobre el desarrollo motor. Investigaciones recientes enfatizan la importancia de la presencia del padre, tanto por su efecto directo en la interacción con el niño como por el apoyo indirecto que representa para la madre en la crianza (Brazelton ${ }^{8}$, 1988).

El hecho que el desarrollo infantil se vea afectado negativamente por la presencia simultánea de factores de riesgo en etapas tan tempranas plantea inquietudes respecto de la evolución del desarrollo en etapas posteriores. Es altamente probable que estos niños continúen su desarrollo en ambientes desventajados, con oportunidades de educación parvularia y escolar también deficitarias. Los desafíos deben orientarse a disminuir las condiciones de riesgo de estos niños, a identificar los factores de resiliencia y apoyar, a través de programas comunitarios, el fortalecimiento de los factores que protejan el desarrollo infantil.

for Early Child Development, 1975.

10. CRAVIOTTO, J. \& DE LICARDIE, E. Environmental correlates of severe clinical malnutrition and language development in survivors from kwashiorkor or marasmus. In: Pan American Health Organization. Nutrition: the nervous system and behavior. Washington, DC, 1972. (Scientific Publication, 251).

11. CRAVIOTTO, J. \& DE LICARDIE, E. Microenvironmental factors in severe protein-energy malnutrition. In: Scrimshow, N.S. \& Behar, M. ed. Nutrition and agricultural development: significance and potential for the tropics. New York Plenum, 1976.

12. DE ANDRACA, I.; COBO, C.; RIVERA, F.; PIZARRO, F. Evaluación de la inteligencia a través de formas cortas del WAIS para grupos de población de nivel socioeconómico bajo. Rev. Saúde Pública, 27: 334-9, 1993.

13. DEVINS, G.M. \& ORME, C.M. Center for epidemiologic studies depression scale. In: Keysas, D.J. ed. Test critique II. Kansas City. Test Corporation of America, 1985. p. 144-60.

14. DOBBING, J. Vulnerable periods in developing brain. In: Davison, A. N. \& Dobbing, J. ed. Applied neurochemestry. Oxford, Blackwell, 1968. p. 287-316.

15. FENDRICH, M.; WARNER. V.; WEISSMAN, M. Family risk factors, parental depression and psychopathology of offspring. Dev. Psychol., 26: 40-50, 1990.

16. GREENSPAN, S. Developmental morbidity in infants in multirisk families. Public Health Rep., 97: 16 - 23, 1982.

17. HOROWITZ, F.D. Using developmental theory to guide the 
search for the effects of biological risk factors on the development of children. Am. Clin. Nutr., 50 (Suppl.): 589$95,1989$.

18. HUSTON, A.C.; MCLOYD, V.C.; GARCIA COLL, C. Children in poverty: issues in contemporary research. Child Dev., 65: 275-82, 1994.

19. KOPP, C.B. \& MCCALL, R.B. Predicting later mental performance for normal, at risk and handicapped infants. In: Baltes, P.B. \& Brim, O.G. ed. Life span development and behavior. New York, Academic Press, 1982. V. 4, p. 33-60.

20. KOPP, C.B. \& KALER, S.R. Risk in infancy: origins and implications. Am. Psychol., 44: 224-30, 1989.

21. LEE, H. S. \& BARRATT, M. Cognitive development of preterm low birth weight children at 5 to 8 years old. J. Dev Beh Ped., 14: 242-9, 1993

22. LOZOFF, B.; PARK, A.M.; RADAN, A.E.; WOLF, A.W. Using the HOME inventory with infants in Costa Rica. Int J. Beh. Dev., 18: 277-95, 1995.

23. LYONS-RUTH K.; CONNELL, D.B.; GRUNEBAUM, H.U. Infants at social risk: maternal depression and family support services as mediators of infant development and security of attachment. Child Dev., 61: 85-98, 1991.

24. NATIONAL CENTER FOR HEALTH STATISTICS Centers For Disease Control. NCHS growth curves for children, birth -18 years. Washington D.C.V.S Government Printing Office, 1978. (Series 11, 165. DHEW publication. (PHS) 78. 1650)

25. POLLITT, E. A critical view of three decades of research on the effects of chronic energy malnutrition on behavioral development. In: Shürch, B. \& Scrimshaw, N. ed. Chronic energy deficiency: consequences and related issues. Lausane IDCG , 1988. p. 77-93.

26. POLLITT, E. A. developmental view of cognition in the undernourished child. Nestlé Foundation Annual Report, p. $88-105.1994$

27. RUTTER, M. Protective factors in children responses to stress and disadventage. In: Kent, M.W. \& Rolf, J.E. ed. Primary prevention in psychopathology. Hanover, University Press of New England, 1979. p. 49-74,

28. SAMEROFF, A. J. Environmental context of child development. J. Ped., 109 (Suppl): 192-200, 1986.

29. SAMEROFF, A.J.; SEIFER, R.; BAROCAS, P.B.; ZACK, M.; GREENSPAN, S. IQ scores for 4-year-old children: social environmental risk factors. Pediatrics, 79: 343-50, 1987.

30. SAMEROFF, A.J.; SEIFER, R.; BALDWIN, A.; BALDWIN, C. Stability of intelligence from preschool to adolescence: the influence of social and family risk factors. Child Dev., 64: 80-97, 1993.

31. SMITH, A.E.A. \& KNIGHT-JONES, E.B. The abilities of very low birthweight children and their classroom controls. Dev. Med. Child Neur, 32: 590-601, 1990.

32. WACHS, T.D. \& GANDOUR M.J. Temperament, environment and six-month cognitive-intellectual development: a test for organismic specificity hypothesis. Int. J. Beh. Dev., 6: 861-7, 1983.

33. WASIK, B.H.; BRYANT, D.M.; LYONS, C.M. Home visiting procedures for helping families. London, Sage Publications, 1991. p. 45-68

34. YOULTON, A. \& VALENZUELA, J. Patrón de crecimiento en estatura y peso de 0 a 17 años y de cicunsferencia craneana de 0 a 2 años en niños de estrato socioeconómico medio-alto y alto de Santiago: comparación con el crecimiento de niños de estrato medio-bajo y bajo del area Norte de Santiago, 1990. Documento de la Rama de Endocrinología y Genética de la Soc. Chilena de Pediatría.

35. ZUCKERMAN, B.S. \& BEARDSLEE, W.R. Maternal depression: a concern for pediatricians. Pediatrics, 79:110-7, 1987. 